\title{
Diretrizes para avaliação e tratamento fisioterapêutico da Síndrome de Pusher: estudo de caso
}

\author{
Guidelines for assessment and physical therapy treatment in Pusher's Syndrome: case report
}

Mariana Callil Voos ${ }^{1,2,3}$, Tatiana de Paula Oliveira ${ }^{1,2}$, Maria Elisa Pimentel Piemonte ${ }^{2,3,4}$

\author{
Estudo desenvolvido no \\ Departamento de Fisioterapia, \\ Fonoaudiologia e Terapia \\ Ocupacional da Faculdade de \\ Medicina da Universidade de São \\ Paulo (USP) - São Paulo (SP), Brasil. \\ ${ }^{1}$ Supervisora de Estágio do Curso \\ de Fisioterapia da USP - São Paulo \\ (SP), Brasil. \\ ${ }^{2}$ Mestre em Neurociências e \\ Comportamento pela USP - São \\ Paulo (SP), Brasil. \\ ${ }^{3}$ Doutora em Neurociências e \\ Comportamento pela USP - São \\ Paulo (SP), Brasil. \\ ${ }^{4}$ Professora do Curso de Fisioterapia \\ da USP - São Paulo (SP), Brasil.
}

\section{ENDERECO PARA CORRESPONDÊNCIA \\ Mariana Callil Voos - Rua \\ Cipotânea, 51 - Cidade \\ Universitária - CEP: 05360-160 \\ - São Paulo (SP), Brasil. E-mail: \\ marivoos@usp.br \\ APRESENTACSÃO \\ out. 2010}

\section{ACEITO PARA PUBLICAÇ̃̃O}

abr. 2011

\section{FONTE DE FINANCIAMENTO nenhuma}

\section{CONFLITO DE INTERESSE nada a declarar}

RESUMO: A Síndrome de Pusher (SP) é uma alteração perceptual presente em 10\% dos casos de hemiparesia causada por Acidente Vascular Encefálico (AVE). Caracterizase por queda para o lado parético, comportamento de empurrar-se para o lado acometido com o hemicorpo não parético e resistência à correção externa. Este estudo visou descrever a avaliação, o tratamento e a evolução de uma paciente com hemiparesia à esquerda e SP, devido a um AVE no hemisfério cerebral direito. Seis meses após a lesão, a paciente realizou testes perceptuais (do desenho da figura humana, comportamental de inatenção, escala de avaliação do sintoma de empurrar, miniexame do estado mental) e de desempenho funcional (escala de avaliação postural para pacientes com AVE, teste de função manual de Jebsen-Taylor, medida de independência funcional e índice de Barthel) e iniciou fisioterapia duas vezes por semana. Cada sessão teve 3 partes de 20 minutos: estimulação sensorial, treino motor e integração sensório-motora. Após o tratamento a paciente foi reavaliada e apresentou melhora em todas as escalas. A de avaliação do sintoma de empurrar e o teste de função manual de Jebsen-Taylor foram os que registraram maiores percentuais de melhora, $79 \%$ e $46 \%$, respectivamente. O protocolo utilizado, mesmo tendo sido iniciado seis meses após a lesão, proporcionou melhora perceptual e funcional, o que sugere a importância da fisioterapia na recuperação da SP.

Descritores: transtornos da percepção; fisioterapia; equilíbrio postural.

ABSTRACT: The Pusher's Syndrome (PS) is a perceptual disorder that occurs in $10 \%$ of the cases of hemiparesis caused by stroke. It is characterized by falling to the paretic side, pushing to the paretic side with the non-paretic side and resisting to external correction. The present study aimed to describe the assessment, treatment and clinical evolution of a patient with left paresis and PS, caused by a stroke on the right hemisphere. Six months after the lesion, the patient was submitted to perceptual (human figure drawing test, behavioral inattention test, scale for contraversive pushing, minimental state examination) and functional performance tests (postural assessment stroke scale, Jebsen-Taylor hand function test, functional independence measure and Barthel index) and started physical therapy twice a week. Each session consisted of 3 parts of 20 minutes: sensory stimulation, motor training and sensorymotor integration. After six months, the patient showed improvement in all scales. The scale for contraversive pushing and the Jebsen-Taylor hand function test showed the highest percentages of improvement, $79 \%$ and $46 \%$, respectively. Besides having been started six months after the lesion, the protocol of the present study contributed to the improvement of perception and functional performance. These findings suggest the importance of the physical therapy treatment in the recovery of individuals with PS.

KEYwords: perceptual disorders; physical therapy; postural balance. 


\section{INTRODUCÃO}

A doença cerebrovascular persiste como importante causa de incapacidade funcional no mundo todo ${ }^{1}$. A hemiparesia, frequente nesses casos, decorre da lesão dos neurônios motores superiores, que controlam os músculos contralesionais, e gera mudanças de equilíbrio e incapacidade funcional ${ }^{2}$. Além do controle motor, alterações de atenção e percepção contribuem para a instabilidade postural e costumam ser mais frequentes após lesão no hemisfério direito ${ }^{3,4}$. Dentre as diferenças percepto-motoras ocorridas após um AVE, a Síndrome de Pusher (SP), presente em cerca de $10 \%$ dos casos $^{5}$, é uma das mais incapacitantes ${ }^{6}$.

A SP é caracterizada por queda para o lado parético, tendência de se empurrar com o lado não parético para o lado parético e resistência à correção postural externa ${ }^{6}$. É comum após lesão póstero-lateral do tálamo ${ }^{7,8}$, que pode acarretar interpretação errônea da verticalidade $^{6,9}$.

Se fixados a uma cadeira inclinável no plano frontal, sem informação visual, indivíduos com SP referem estar com alinhamento corporal adequado quando, na verdade, estão inclinados lateralmente em cerca de 20 graus. Entretanto, a percepção de verticalidade visual não está alterada, já que conseguem posicionar corretamente um feixe de luz e objetos na posição vertical, mesmo quando mantêm o tronco desalinhado9.

Há poucos estudos sobre a fisioterapia para indivíduos com SP. Muitos não descrevem o tratamento, utilizando apenas termos como "fisioterapia de acordo com as necessidades de cada paciente ${ }^{\prime \prime 5,10} \mathrm{e}$ "fisioterapia respiratória e motora"11.

Dentre as pesquisas que mencionam a abordagem fisioterapêutica, são descritas como condutas eficazes: conscientização do desalinhamento postural ${ }^{12}$; treino do uso de elementos no ambiente, como pistas para auxiliar no alinhamento corporal ${ }^{11-14}$; estimulação somatossensorial para compensar a dificuldade de integração dos sistemas visual e vestibular ${ }^{14}$; treino dos movimentos necessários para atingir e manter a postura alinhada ${ }^{12-15}$; manutenção da postura alinhada durante a realização de uma tarefa associada ${ }^{12}$; transferência de peso nas posturas sentada e em pé, em diversas direções ${ }^{11}$; transferência da posição sentada para ortostatismo, utilizando o membro não parético como apoio $^{11}$; e treino de marcha ${ }^{11}$.

A maioria dos estudos propõe um mês de acompanhamento ${ }^{4-7,11-13}$, porém não há consenso na literatura do tempo ideal de tratamento e, em geral, indivíduos com SP têm recuperação mais longa do que indivíduos com AVE sem essa alteração $^{5}$. Além disso, a presença da heminegligência associada à SP parece influenciar no prognóstico, aumentando o tempo de recuperação ${ }^{4,5,10}$.

Não foram encontrados estudos a respeito da eficácia de condutas em pacientes que mantiveram o quadro de SP após quatro ou mais meses decorridos da lesão. Foram verificadas pesquisas apenas com pacientes na fase aguda ou sub-aguda após o AVE ${ }^{4,10-15}$, quando é frequente a recuperação espontânea de parte das limitações apresentadas, o que deixa dúvidas sobre qual seria a melhor forma de programar o tratamento, sobretudo para pacientes crônicos.

O estudo visou descrever a avaliação e o tratamento de uma paciente com hemiparesia à esquerda e SP, decorrentes de lesão vascular hemorrágica crônica no hemisfério direito.

\section{METODOLOGIA}

Foi voluntária da pesquisa uma paciente do sexo feminino, 65 anos, destra, dona de casa, com diagnóstico médico de AVE hemorrágico em núcleo lentiforme, cápsula interna e tálamo, ocorrido há seis meses (Figura 1). O filho, responsável por ela, assinou o termo de Consentimento Livre e Esclarecido, aprovado pelo Comitê de Ética, permitindo a participação neste estudo paralelamente ao tratamento fisioterapêutico. A paciente encontrava-se medicada com fenitoína (100 mg/dia), gibenclamida (5 mg/dia), hidroclorotiazida (50 mg/dia) e captopril (50 mg/dia). Referiu como antecedentes pessoais tabagismo, hipertensão arterial sistêmica e diabetes mellitus, e não realizou outras terapias no período estudado.

\section{Avaliação}

A paciente era dependente para vestuário, higiene pessoal, locomoção (utilizava cadeira de rodas) e semidependente para alimentação (quando recebia o alimento cortado conseguia levá-lo à boca com membro superior direito). No Índice de Barthel (IB) ${ }^{16}$ e na Medida de Independência Funcional (MIF) ${ }^{14}$, que avaliam atividades de vida diária, recebeu, respectivamente, 10/100 e 36/126. No Mini Exame do Estado Mental $\left(\right.$ MEEM) ${ }^{17}$ obteve $17 / 30$ pontos.

Conseguia rolar para o decúbito lateral esquerdo sem auxílio, porém era incapaz de assumir ou manter o decúbito lateral direito sozinha. Não conseguia assumir ou manter-se em sedestação, caindo para a esquerda. Recebeu pontuação 6/36 na escala de avaliação postural (EAP) para pacientes com $\mathrm{AVE}^{18}$, que detecta a capacidade de manter posturas como sedestação e ortostatismo. Na escala de avaliação do sintoma de empurrar (EASE) ${ }^{12}$, que diagnostica e quantifica a SP (e pontua indivíduos sem alterações posturais com zero), recebeu $4,75 / 6,00$ pontos.

$\mathrm{Na}$ avaliação fisioterapêutica inicial não foi observada alteração na sensibilidade tátil, dolorosa, térmica, proprioceptiva, visual e auditiva ${ }^{19}$. Havia espasticidade à esquerda em inversores e flexores plantares de tornozelo, extensores de joelho, flexores, rotadores mediais e adutores de quadril, adutores de ombro e flexores de cotovelo. Havia fraqueza muscular em membro inferior esquerdo (grau 2 para abdutores de

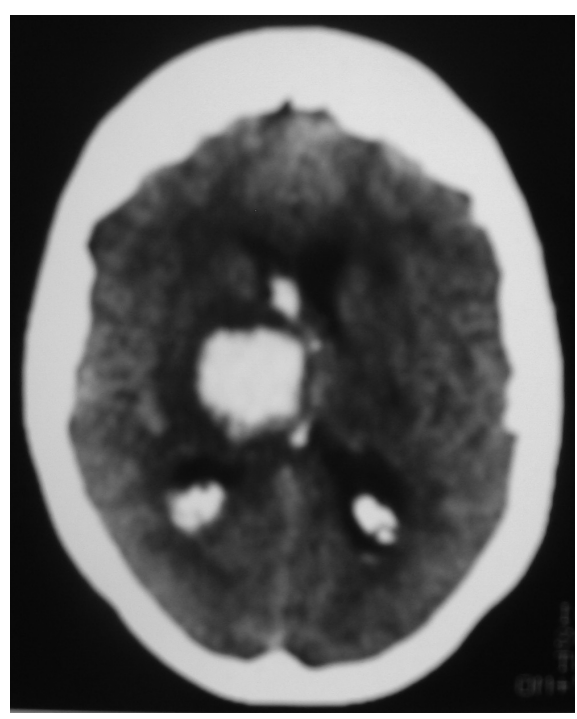

Figura 1. Tomografia computadorizada com lesão hemorrágica em tálamo, núcleo lentiforme e cápsula interna à direita 
quadril, rotadores laterais de quadril e eversores de tornozelo e grau 3 para os demais grupos musculares do membro inferior), em tronco (grau 3 para flexores, extensores, inclinadores laterais e rotadores à esquerda) e em membro superior esquerdo (grau 2 para flexores, extensores, rotadores laterais e mediais de ombro e grau 3 para demais grupos musculares)

No teste de função manual de JebsenTaylor $(\mathrm{FMJT})^{20}$, que avalia a agilidade, o lado direito recebeu 90 (desempenho normal) e o esquerdo recebeu 584 pontos (todas as tarefas acima do tempo máximo). A paciente referiu que, antes da lesão, apresentava habilidade para desenho, mas, embora a força e a coordenação do membro superior direito não tivessem sofrido alterações, suas criações não eram mais como antes.

A paciente levou desenhos feitos antes da lesão, comparados aos realizados em terapia. Ela não conseguia mais desenhar mãos e braços com a mesma habilidade. O traçado do lado esquerdo do corpo era menos preciso e às vezes desproporcional. A escala do desenho da figura humana $(E D F H)^{21}$, que gradua entre zero e 30 a capacidade de reproduzir graficamente a figura humana, foi pontuada como zero (Figura 2).

No protocolo de inatenção visuoespacial, utilizado para detectar heminegligência ${ }^{22}$, foi verificada alteração do desempenho na secção de linhas horizontais $(\mathrm{SLH})$, com desvio de $5 \mathrm{~cm}$ para a direita, e no cancelamento de estrelas

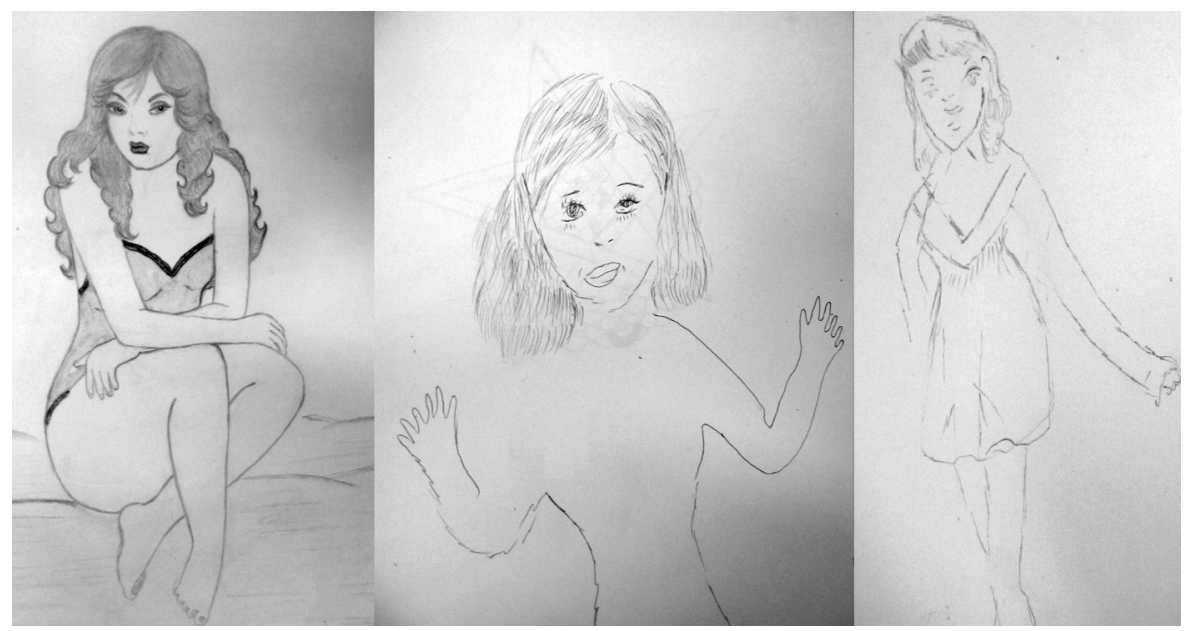

Figura 2. Desenhos realizados pela paciente antes da lesão (esquerda), quando iniciou fisioterapia (centro) e após seis meses de tratamento (direita)
(CE), com 13 estrelas omitidas à esquerda e 4 à direita.

Associando os achados do desenho da figura humana aos do protocolo de inatenção visuoespacial, verificamos a incapacidade de processar adequadamente estímulos do hemicampo esquerdo. Ou seja, a paciente apresentava um quadro de SP associado à heminegligência que, segundo dados da literatura, tem recuperação mais lenta e pior prognóstico funcional $\left.\right|^{4,5,10}$.

\section{Tratamento}

A paciente recebeu sessões de uma hora, duas vezes por semana, no ambulatório de fisioterapia da Universidade de São Paulo e orientações semanais para realização de exercícios em casa. Os objetivos foram melhorar as trocas de decúbito, o equilíbrio e o alinhamento em sedestação e a estabilidade do ombro esquerdo. A conduta consistiu em 3 partes, com 20 minutos cada, realizadas em todas as sessões.

Na parte 1 foram realizados exercícios para estimulação sensorial - estímulos visuais ajudam a corrigir o desalinhamento postural ${ }^{13}$. A realimentação visual sobre a postura vertical correta, realizada com auxílio de um espelho, auxilia na conscientização do alinhamento $^{12}$. Por isso, a terapeuta solicitou espaço e utilizou figuras do ambiente como pistas para auxiliar no alinhamento corporal ${ }^{11-14}$. Também foram usadas a realimentação tátil e somatossensorial a descrição de elementos do corpo e do para compensar a dificuldade de integrar informações visuais, somestésicas e vestibulares ${ }^{6,8,14}$.

Na parte 2 houve treino motor, com mudanças de decúbito, transferência cadeira de rodas-tablado ${ }^{14}$, alinhamento e funcionalidade em sedestação, com e sem a realização associada de movimentos dos membros superiores ${ }^{12-15}$ e transferência de peso em diversas direções ${ }^{11}$. Também foi estabilizada a cintura escapular e foram fortalecidos os músculos do tronco, membros superiores e inferiores ${ }^{23}$.

Na parte 3 foram realizados exercícios para favorecer a integração sensóriomotora, como passar objetos do lado direito para o lado esquerdo do tablado (e vice-versa $)^{9,24}$, usar camiseta com um risco vertical e manter o risco paralelo a outra reta vertical traçada no espelho ${ }^{24} \mathrm{e}$ iluminar com uma lanterna fixa, ora no ombro direito, ora no esquerdo, ora no topo na cabeça, com alvos em diferentes locais do espaço ${ }^{24}$.

\section{RESULTADOS}

Após seis meses de tratamento, a paciente passou a utilizar o membro superior esquerdo em algumas atividades de vida diária, como se vestir, tomar banho e se alimentar com auxílio. Tornou-se independente para pentear os cabelos e escovar os dentes. Passou a manter-se sentada sem apoio, sem cair para o lado esquerdo e realizar as transferências da cadeira para a cama e vice-versa (Figura 3), necessitando apenas de supervisão e informações espaciais complementares para se alinhar corretamente na cadeira (como, por exemplo, "vá mais para frente", "mais para direita", etc.) Recebeu 50/100 no IB, 67/126 na MIF e 24/30 no MEEM, com melhora de, respectivamente, 40, 24 e $23 \%$.

A paciente adquiriu capacidade para assumir e manter decúbitos laterais e sedestação sem auxílio. A tendência de queda para a esquerda passou a ocorrer apenas quando era acrescentada uma distração, incluindo tarefas de enumeração, matemática e soletrar palavras. A EAP foi pontuada em 19/36, com melhora de $36 \%$. A EASE foi reaplicada e pontuada como 0,00/6,00 (ausência de SP), com melhora de $79 \%$. 


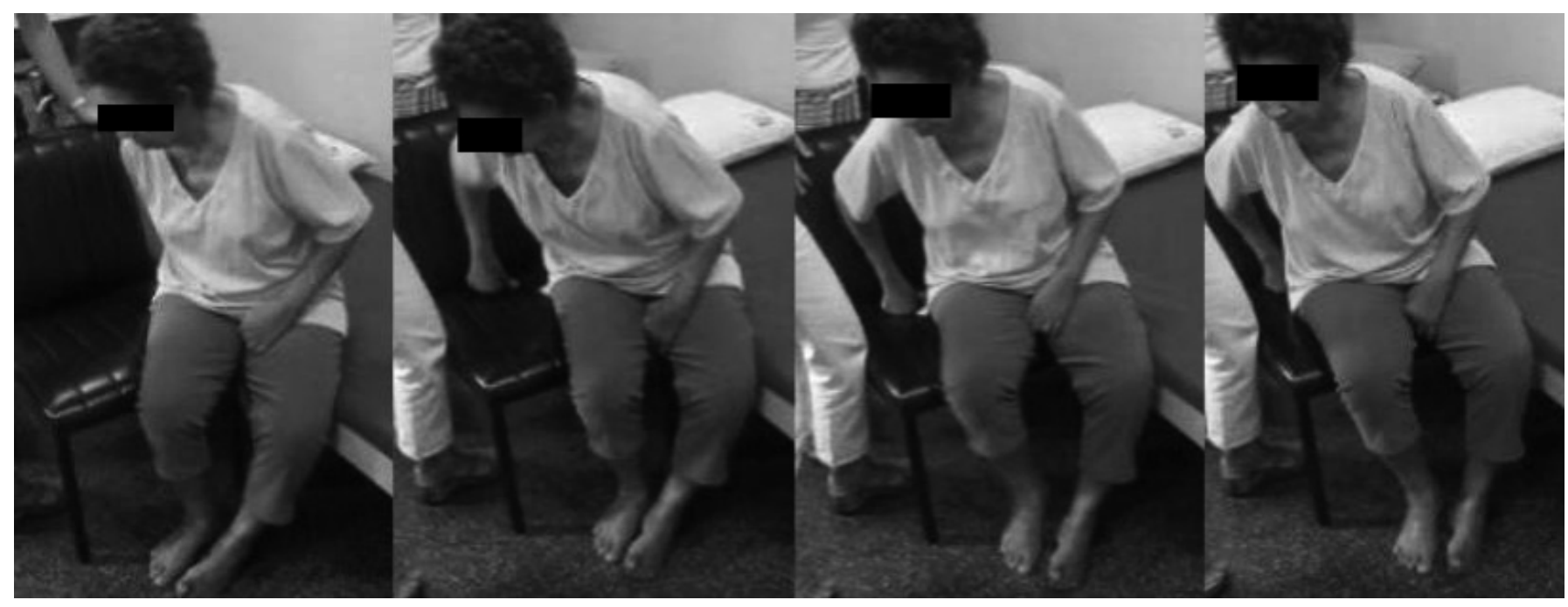

Figura 3. Transferência do tablado para a cadeira após o tratamento

Tabela 1. Melhora apresentada nas escalas após o tratamento

\begin{tabular}{lccccccccc}
\hline & IB & MIF & MEEM & EAP & EASE & FMJT & EDFH & SLH & CE \\
\hline Inicial & 10,00 & 36,00 & 17,00 & 6,00 & 4,75 & $90,00 \mathrm{D} / 560,00 \mathrm{E}$ & 0,00 & 5,00 & $4,00 \mathrm{D} / 13,00 \mathrm{E}$ \\
Final & 50 & 67 & 24 & 19 & 0 & $80 \mathrm{D} / 301 \mathrm{E}$ & 9 & 2 & $2 \mathrm{D} / 8 \mathrm{E}$ \\
Melhora (\%) & 40 & 24 & 23 & 36 & 79 & $2 \mathrm{D} / 46 \mathrm{E}$ & 30 & 20 & $14 \mathrm{D} / 36 \mathrm{E}$ \\
Total & $0-100$ & $18-126$ & $0-30$ & $0-36$ & $6-0$ & $560-50$ & $0-30$ & $15-0$ & $14-0 \mathrm{D} / 14-0 \mathrm{E}$ \\
\hline
\end{tabular}

IB: Índice de Barthel; MIF: Medida de Independência Funcional; MEEM: Mini Exame do Estado Mental; EAP: Escala de Avaliação Postural; EASE: Escala de Avaliação do Sintoma de Empurrar; FMJT: Função Manual de Jebsen-Taylor; EDFH: Escala de Desenho da Figura Humana; SLH: Secção de linhas horizontais; CE: Cancelamento de estrelas; Inicial: Pontuação antes do tratamento fisioterapêutico; Final: Pontuação depois do tratamento fisioterapêutico; Melhora: Diferença entre final e inicial em porcentagem; Total: Pior-melhor desempenho possível na escala

Houve melhora também na estabilidade do ombro esquerdo e na força do membro superior esquerdo (grau 3 para flexores, extensores, rotadores laterais e mediais de ombro e 4 para os demais grupos musculares). Observou-se manutenção do quadro de tônus muscular. Registrou-se avanço da força muscular de abdutores e rotadores laterais do quadril (de 2 para 3).

O membro superior passou a ser mais usado nas atividades funcionais, sendo necessário menos estímulo por parte de familiares e da fisioterapeuta. No FMJT, o membro superior direito recebeu 80 , o que significa desempenho normal, e o membro superior esquerdo, ainda alterado, porém mais funcional, recebeu 301 pontos (melhora de $2 \%$ para o lado direito e de $46 \%$ para o lado esquerdo). Mesmo sem nenhum treinamento específico para a habilidade de desenhar, essa capacidade foi parcialmente recuperada. A EDFH foi pontuada como 9/30, com ganho de $30 \%$ no desempenho. No protocolo de inatenção visuoespacial também diminuiu a alteração do desempenho na SLH (desvio para a direita de $2 \mathrm{~cm}$, com melhora de 20\%) e no CE (8 estrelas esquecidas à esquerda e 2 à direita, com melhora de $14 \%$ à direita e $36 \%$ à esquerda).

Todos os resultados e os respectivos percentuais de melhora estão representados na Tabela 1.

\section{DISCUSSÃO}

Na parte 1 do protocolo foram realizados exercícios para estimulação sensorial, pois o aumento de informações sensoriais, por meio da estimulação de aferências íntegras, pode auxiliar no controle do tronco ${ }^{24}$ e compensar a deficiência vestibular, presente na $\mathrm{SP}^{9}$. Mesmo com o resultado normal do teste clínico de propriocepção, realizado por meio da solicitação da descrição da posição do hálux e do indicador da paciente, após mobilização do examinador, é possível a existência de alteração do processamento da informação somestésica para o alinhamento postural ${ }^{12}$.

O treino de controle de tronco, abordado na parte 2, pode melhorar a independência funcional, comumente prejudicada na $\mathrm{SP}^{4}$, e proporcionar postura mais estável $^{14}$ e melhor processamento sensorial ${ }^{3}$. De fato, apesar de a paciente ainda precisar de auxílio em atividades diárias ao final do tratamento, ocorreu melhora funcional ( $40 \%$ no IB e $24 \%$ na MIF) e de controle de tronco (melhora de 36\% na EAP).

Na parte 3 foram realizados exercícios para favorecer a integração sensório-motora, pois há evidências de que estímulos auditivos, mnêmicos, táteis e proprioceptivos podem facilitar a alocação da atenção para o lado esquerdo e, com isso, melhorar o desempenho em tarefas que requerem integração sensório-motora ${ }^{3,24}$. A estimulação visual por meio da observação ou movimentação de objetos de um lado para outro e o uso de pontos de 
fixação no espaço, para a manutenção do alinhamento correto, também mostraram bons resultados em estudos prévios ${ }^{3,9} \mathrm{e}$ na presente pesquisa.

Alterações de alinhamento e instabilidade postural e perda do esquema corporal são mais frequentes após lesões à direita ${ }^{3,5,9,25}$. Indivíduos com déficit atencional tendem a apresentar instabilidade postural, principalmente quando processam estímulos simultâneos ${ }^{25}$. A associação da inatenção com a SP pode ter sido um dos fatores que contribuiu para o agravamento do quadro da paciente, como descrito por Danells et al. ${ }^{5}$ Embora o protocolo utilizado neste estudo tenha melhorado os sintomas de SP, também houve avanço do quadro de heminegligência, verificado pelo maior ganho percentual do desempenho do lado esquerdo no FMJT e no $\mathrm{CE}$, diminuição do desvio no teste de SLH e melhora do desenho da figura humana.

Quanto à força muscular, SantosPontelli et al. ${ }^{15}$ sugeriram que o grau de hemiparesia não influencia no prognóstico e na gravidade da SP, já que, assim como neste estudo, verificaram a manutenção de prejuízos motores (grau de força muscular 3 no membro superior e 4 no inferior esquerdo), embora tenham observado a resolução total da SP após o tratamento fisioterapêutico. Todavia, Cecatto \& Almeida ${ }^{14}$ relataram melhora importante do quadro motor de um paciente com SP e manutenção parcial do sintoma de empurrar-se para o lado acometido (pontuação inicial de 4,50 e final de 0,75).

Como os casos descritos por SantosPontelli et al. ${ }^{15}$ e por Cecatto \& Almeida ${ }^{14}$ encontravam-se na fase aguda após o AVE, é difícil separar as aquisições decorrentes da recuperação espontânea das proporcionadas pelo tratamento fisioterapêutico. Por outro lado, no presente estudo, as aquisições foram observadas na fase crônica, o que sugere que tenham sido resultado do tratamento. Além disso, as propostas Dos autores enfatizaram o treino motor e funcional (compatível com a parte 2 do protocolo do presente estudo), reservando menor espaço para condutas mais específicas de estimulação sensorial e sensório-motora, as quais, possivelmente, contribuíram para a grande melhora da EASE (79\%), escala com maior percentual de melhora do presente estudo.

Como limitações da pesquisa, podemos citar a impossibilidade de realização de uma avaliação após seis meses decorridos da alta ambulatorial para verificação da retenção dos ganhos adquiridos logo após a fisioterapia devido à mudança de cidade da paciente e à ausência de testes quantitativos de sensibilidade, que possibilitariam melhor detecção da eficácia das partes 1 e 3 do protocolo.

É importante ressaltar que, no presente estudo, o início tardio da fisioterapia não foi intencional, mas fruto da dificuldade que alguns pacientes ainda têm em iniciar o tratamento no sistema público de saúde no Brasil, enfrentando filas de espera. Novas pesquisas são necessárias para a sistematização de condutas cada vez mais adequadas para indivíduos com SP, a fim de que as disfunções sejam menores ao final do processo de reabilitação.

\section{CONCLUSÃO}

Após seis meses de tratamento fisioterapêutico com estimulação sensorial, estimulação motora e integração sensóriomotora, a paciente apresentou melhora perceptual e funcional, o que mostra que a fisioterapia, mesmo quando iniciada seis meses após o AVE, pode minimizar as disfunções da SP.

\section{REFERÊNCIAS}

1. Lavados PM, Hennis AJ, Fernandes JG, Medina MT, Legetic B, Hoppe A, et al. Stroke epidemiology, prevention, and management strategies at a regional level: Latin America and the Caribbean. Lancet Neurol. 2007;6(4):362-72.

2. Tyson SF, Hanley M, Chillala J, Selley A, Tallis RC. Balance disability after stroke. Phys Ther. 2006;86(1):30-8.

3. Voos MC, Ribeiro do Valle LE. Estudo comparativo entre a relação do hemisfério acometido no acidente vascular encefálico e a evolução funcional em indivíduos destros. Rev Bras Fisioter. 2008;12(2):113-20.

4. Lafosse C, Kerckhofs E, Troch M, Vereeck L, Van Hoydonck G, Moeremans M, et al. Contraversive pushing and inattention of the contralesional hemispace. J Clin Exp Neuropsychol. 2005;27(4):460-84.

5. Danells CJ, Black SE, Gladstone DJ, Mcllroy WE. Poststroke "pushing": natural history and relationship to motor and functional recovery. Stroke. 2004;35(12):2873-8.

6. Baccini M, Paci M, Nannetti L, Biricolti C, Rinaldi LA. Scale for contraversive pushing: cutoff scores for diagnosing "pusher behavior" and construct validity. Phys Ther. 2008;88(8):947-55.

7. Johannsen L, Broetz D, Naegele T, Karnath HO. "Pusher syndrome" following cortical lesions that spare the thalamus. J Neurol. 2006;253(4):455-63.

8. Ticini LF, Klose U, Nägele T, Karnath HO. Perfusion imaging in Pusher syndrome to investigate the neural substrates involved in controlling upright body position. PLoS One. 2009;4(5):e5737.

9. Pérennou DA, Mazibrada G, Chauvineau V, Greenwood R, Rothwell J, Gresty MA, et al. Lateropulsion, pushing and verticality perception in hemisphere stroke: a causal relationship?. Brain. 2008;131(9):2401-13.

10. Santos-Pontelli TE, Pontes-Neto OM, Colafêmina JF, de Araujo DB, Santos AC, Leite JP. Contraversive pushing in non-stroke patients. J Neurol. 2004;251(11):1324-8.

11. Paci M, Nannetti L. Physiotherapy for pusher behaviour in a patient with post-stroke hemiplegia. J Rehabil Med. 2004;36(4):183-5.

12. Karnath $\mathrm{HO}$, Broetz D. Understanding and treating "pusher syndrome". Phys Ther. 2003;83(12):1119-25. 


\section{Referências (cont.)}

13. Broetz D, Johannsen L, Karnath HO. Time course of 'pusher syndrome' under visual feedback treatment. Physiother Res Int. 2004;9(3):138-43.

14. Cecatto RB, Almeida Cl. Pusher syndrome after cerebrovascular accident: case report. Acta Fisiátrica. 2008;15(3):195-201.

15. Santos-Pontelli TE, Pontes-Neto OM, Colafêmina JF, Araújo DB, Santos AC, Leite JP. Pushing behavior and hemiparesis: which is critical for functional recovery in pusher patients? Case report. Arq Neuropsiquiatr. 2007;65(2B):536-9.

16. Mahoney FI, Barthel DW. Functional evaluation: the barthel index. Md State Med J. 1965;14:61-5.

17. Folstein MF, Folstein SE, McHugh PR. "Mini-mental state". A practical method for grading the cognitive state of patients for clinician. J Psychiatr Res. 1975; 12(3):189-98.

18. Benaim C, Pérennou DA, Villy J, Rousseaux M, Pelissier JY. Validation of a standardized assessment of postural control in stroke patients: the Postural Assessment Scale for Stroke Patients (PASS). Stroke. 1999;30(9):1862-8.
19. Mutarelli EG. Propedêutica neurológica: do sintoma ao diagnóstico. São Paulo: Sarvier, 2000.

20. Jebsen RH, Taylor N, Trieschmann RB, Trotter MJ, Howard LA. An objective and standardized test of hand function. Arch Phys Med Rehabil. 1969;50(6):311-9.

21. Sisto FF. Desenho da figura humana - Escala Sisto. Manual. São Paulo: Vetor, 2005.

22. Wilson B, Cockburn J, Halligan P. Development of a behavioral test of visuospatial neglect. Arch Phys Med Rehabil. 1987;68(2):98-102.

23. Flansbjer UB, Miller M, Downham D, Lexell J. Progressive resistance training after stroke: effects on muscle strength, muscle tone, gait performance and perceived participation. J Rehabil Med. 2008;40(1):42-8.

24. Shumway-Cook A, Woollacott M. Controle motor: teoria e aplicações práticas.Barueri: Manole; 2003.

25. Honoré J, Saj A, Bernati T, Rousseaux M. The pusher syndrome reverses the orienting bias caused by spatial neglect. Neuropsychologia. 2009;47(3):634-8. 